BESANÇON

An explicit formula for the Hilbert symbol in a multidimensional local field

T.B. BELIAEVA

S.V. VOSTOKOV 


\title{
AN EXPLICIT FORMULA FOR THE HILBERT SYMBOL IN A MULTIDIMENSIONAL LOCAL FIELD
}

\author{
T.B. Beliaeva, S.V. Vostokov
}

\section{INTRODUCTION}

The object of this paper to expand the results of the paper [1]. In it was constructed the explicit formula for the Hilbert symbol for a mutidimensional local field of characteristic 0 with the residue field of characteristic $p$, where $p$ is an odd prime number. In this paper we consider the case of $p=2$.

Let $F$ be an $n$-dimensional local field, i.e. a sequence of fields $k_{0}, k_{1}, \ldots, k_{n}=F$ such that:

a) $k_{0}$ is finite;

b) $\forall i=1, \ldots, n$ the field $k_{i}$ is a complete discrete valuated field with the residue field $k_{i-1}$.

Assume that $F$ contains the group $\mu_{q}$ of $q$-th roots of 1 , where $q=2^{m}$, and let $\zeta$ be a generator of $\mu_{q}$. Then we can define the Hilbert symbol as follows

$$
\begin{gathered}
(,)_{q}: k_{q}^{M}(F) \times F^{*} / F^{* q} \rightarrow \mu_{q}, \\
\left(\left\{\alpha_{1}, \ldots, \alpha_{n}\right\}, \beta\right)_{q}=\sqrt[q]{\beta}^{\psi\left(\left\{\alpha_{1}, \ldots, \alpha_{n}\right\}\right)-1},
\end{gathered}
$$

where $k_{q}^{M}(F)=K_{n}^{M}(F) / K_{n}^{M}(F)^{q}$ and $K_{n}^{M}(F)$ is the $n$-th Milnor's K-group,and

$$
\psi: K_{n}^{M}(F) \longrightarrow \operatorname{Gal}\left(F^{a b} / F\right)
$$

is a canonical reciprocity map on $F$.

The aim of this article is to give an explicit formula for $(,)_{q}$. We construct the map

$$
\begin{gathered}
\Gamma: F^{*} \times \cdots \times F^{*} \rightarrow \mu_{q} \\
\Gamma\left(\alpha_{1}, \ldots, \alpha_{n+1}\right)=\zeta^{\operatorname{tr} \operatorname{res} \Phi W},
\end{gathered}
$$

where tr is a trace operator on the inertia subfield of $F$, res denotes a standard residue of a power series, $W$ is a power series, defined by the expansion of $\zeta$ in power series on the local parameters of the field $F$, and $\Phi$ is defined by the similar expansions of the elements $\alpha_{i}$.

We will prove the following theorems given in the case $p \neq 2$ in the paper [1]:

This work was partially supported by the Réseau Formation-Recherche of the Franch Ministry of the National Education.

The second author aknowledges support by the Russian Foundation of fundamential Research Grant 97-01-0058-a. 
Theorem 1. The map $\Gamma$ has the following properties:

a) multiplicativity at all the arguments;

b) anticommutativity, i.e. $\Gamma\left(\ldots, \alpha_{i}, \alpha_{i+1}, \ldots\right)=\Gamma\left(\ldots, \alpha_{i+1}, \alpha_{i}, \ldots\right)^{-1}$;

c) proportionality, i.e. $\Gamma\left(\ldots, \alpha_{i}, \alpha_{i+1}, \ldots\right)=1$ when $\alpha_{i}+\alpha_{i+1}=0$;

d) symbol property, i.e. $\Gamma\left(\ldots, \alpha_{i}, \alpha_{i+1}, \ldots\right)=1$ when $\alpha_{i}+\alpha_{i+1}=1$.

Theorem 2. The map $\Gamma$ is well defined, i.e. it is invariant with respect to any change of variables and independent on the method of expansion of $\alpha_{i}$ and $\zeta$ in power series.

Theorem 3. The pairing

$$
\langle,\rangle_{\Gamma}: k_{q}(F) \times F^{*} / F^{* q} \longrightarrow \mu_{q},
$$

where $k_{q}(F)=K_{n}^{M}(F) / K_{n}^{M}(F)^{q}$, given by the formula

$$
\left\langle\left\{\alpha_{1}, \ldots, \alpha_{n}\right\}, y\right\rangle_{\Gamma}=\Gamma\left(\alpha_{1}, \ldots, \alpha_{n}, y\right),
$$

coincides with the Hilbert symbol $(,)_{q}$.

\section{$\S 1$ Description OF THE MAP $\Gamma$.}

The field $F$ contains (as an isomorphic subfield) a quotient field of the Witt ring $W\left(k_{0}\right)$, which is the inertia subfield of $F$. Let us denote this subfield by $T$, and its ring of integers by $\mathfrak{o}$. Let $t_{1}, \ldots, t_{n-1}$ be local uniformizing elements of the field $F$. We consider the ring $A=\mathfrak{o}\left\{\left\{t_{1}\right\}\right\} \ldots\left\{\left\{t_{n-1}\right\}\right\}$ and define on the ring $A\left\{\left\{t_{n}\right\}\right\}$ the Frobenius operator $\Delta$, wich acts on $t_{i}$ as squaring and leaves coefficients invariant.

Let $\pi$ be a prime element of $F$. Then we can expand $\zeta$ in a series on the exponents of $\pi$ with coefficients in the ring $A$ and replasing $\pi$ by $t_{n}$ obtain series $z\left(t_{n}\right)$ of $A\left(\left(t_{n}\right)\right)$ such that $z(\pi)=\zeta$. In the same way we can obtain the series $\alpha_{i}\left(t_{n}\right)$ for each of the $\alpha_{i}$.

We denote by $W$ the formal power series, given by the same formula as the power series $\chi / s$ from the papers [2.1] and [2.2]. This power series depends only on $z\left(t_{n}\right)$ and has the following property:

$$
\frac{\partial W}{\partial t_{i}} \equiv 0 \quad \bmod \quad q, \quad 1 \leq i \leq n
$$

Define $l(\alpha)$ for any $\alpha \in A\left\{\left\{t_{n}\right\}\right\}$ as follows:

$$
l(\alpha)=\frac{1}{2} \log \alpha^{2-\Delta} .
$$

The function $l(\alpha)$ is well defined because of the obvious congruence:

$$
\alpha^{\Delta} \equiv \alpha^{2} \quad \bmod 2,
$$

and it is easy to prove that $l(\alpha) \in A\left\{\left\{t_{n}\right\}\right\}$ for any $\alpha \in A\left\{\left\{t_{n}\right\}\right\}$. 
Denote the logarithmic derivative $\alpha^{-1} \frac{\partial \alpha}{\partial t_{i}} \forall \alpha \in A\left\{\left\{t_{n}\right\}\right\}$ by $\delta_{i}(\alpha)$, the difference $\delta_{i}(\alpha)-\frac{\partial l(\alpha)}{\partial t_{i}}$ by $\eta_{i}(\alpha), \sum_{i \geq 0} l^{\Delta^{\prime}}(\alpha)$ by $\sigma(\alpha)$ and $\frac{\partial}{\partial t_{i}} \frac{\Delta}{2} \sigma(\alpha) \sigma(\beta)$ by $\nu_{i}(\alpha, \beta)$. We define $\Phi$ by the following formula:

$$
\Phi=\sum_{i=1}^{n+1}(-1)^{n-i+1} l\left(\alpha_{i}\right) D_{i}+\sum_{\substack{i=2, j=1 \\ i>j}}^{n+1} P_{i, j}
$$

where

$$
D_{i}=\left|\begin{array}{ccc}
\delta_{1}\left(\alpha_{1}\right) & \ldots & \delta_{n}\left(\alpha_{1}\right) \\
\ldots & \ldots & \ldots \\
\delta_{1}\left(\alpha_{i-1}\right) & \ldots & \delta_{n}\left(\alpha_{i-1}\right) \\
\eta_{1}\left(\alpha_{i+1}\right) & \ldots & \eta_{n}\left(\alpha_{i+1}\right) \\
\ldots & \ldots & \ldots \\
\eta_{1}\left(\alpha_{n}\right) & \ldots & \eta_{n}\left(\alpha_{n}\right)
\end{array}\right|, P_{i, j}=\left|\begin{array}{ccc}
\delta_{1}\left(\alpha_{1}\right) & \ldots & \delta_{n}\left(\alpha_{1}\right) \\
\ldots & \ldots & \ldots \\
\delta_{1}\left(\alpha_{j-1}\right) & \ldots & \delta_{n}\left(\alpha_{j-1}\right) \\
\nu_{1}\left(\alpha_{j}, \alpha_{i}\right) & \ldots & \nu_{n}\left(\alpha_{j}, \alpha_{i}\right) \\
\eta_{1}\left(\alpha_{j+1}\right) & \ldots & \eta_{n}\left(\alpha_{j+1}\right) \\
\ldots & \ldots & \ldots \\
\eta_{1}\left(\alpha_{n}\right) & \ldots & \eta_{n}\left(\alpha_{n}\right)
\end{array}\right|
$$

Set

$$
\Phi^{(1)}=\sum_{i=1}^{n+1}(-1)^{n-i+1} l\left(\alpha_{i}\right) D_{i}, \quad \Phi^{(2)}=\sum_{\substack{i=2, j=1 \\ i>j}}^{n+1} P_{i, j}, \quad\left(\Phi^{(1)}\right. \text { is the same as }
$$

$\Phi$ in the paper [1]), then we obtain a more simple representation for $\Phi$ :

$$
\Phi=\Phi^{(1)}+\Phi^{(2)} .
$$

\section{$\S 2$ SOME USEFUL DEFINITIONS AND FACTS}

Let $A_{i}$ be the ring o $\left\{\left\{t_{1}\right\}\right\} \ldots\left\{\left\{t_{i-1}\right\}\right\}\left\{\left\{t_{i+1}\right\}\right\} \ldots\left\{\left\{t_{n}\right\}\right\}$. We consider the ArtinHasse function on $A_{i}$ :

$$
\mathcal{E}(\alpha)=\exp \sum_{l=0}^{\infty} \frac{\alpha^{2^{l}}}{2^{l}}, \quad \alpha \in A_{i}
$$

Let $\varphi$ be an extension of the Frobenius operator from $W\left(k_{0}\right)$ to $A_{i}$. Then we can define the generalized Artin-Hasse function on $A_{i}$ :

$$
E_{\varphi}(\alpha)=\exp \sum_{l=0}^{\infty} \frac{\varphi^{l}(\alpha)}{2^{l}}
$$

For the extension of the Frobenius operator $\Delta: t_{i} \longmapsto t_{i}^{2}$ given above we denote the function $E_{\varphi}(\alpha)$ by $E$, i.e.

$$
E(\alpha)=\exp \sum_{l=0}^{\infty} \frac{\alpha^{\Delta^{l}}}{2^{l}}
$$


Obviously, for $\alpha=t_{1}^{i_{1}} \cdots t_{n}^{i_{n}}$, we have

$$
\mathcal{E}(\alpha)=E(\alpha)
$$

Besides, it is easy to prove that the functions $l$ and $E$ are mutually inverse.

If a series $\psi$ is the sum of partial derivatives of some series from the ring $A\left\{\left\{t_{n}\right\}\right\}$ we shall say that

$$
\psi \equiv 0 \bmod \partial .
$$

Lemma 1. If $\Psi \in A\left\{\left\{t_{n}\right\}\right\}$ satisfies the congruence $\Psi \equiv 0 \bmod \partial$, then $\Psi \cdot W \equiv 0 \bmod q$.

Corollary. If $\Phi\left(\alpha_{1}, \ldots, \alpha_{n+1}\right) \equiv 0 \bmod \partial$, then $\Gamma\left(\alpha_{1}, \ldots, \alpha_{n+1}\right)=1$.

Lemma 2. Let the series $\varphi_{1}, \ldots, \varphi_{n-1}$ and $\psi$ be such that $\psi \in A\left\{\left\{t_{n}\right\}\right\}$ and $\frac{\partial \varphi_{i}}{\partial t_{j}} \in A\left\{\left\{t_{n}\right\}\right\} \forall 1 \leq i \leq n-1,1 \leq j \leq n$, then the series

$$
D=\left|\begin{array}{c}
\frac{\partial \psi}{\partial t_{1}} \ldots \frac{\partial \psi}{\partial t_{n}} \\
\frac{\partial \varphi_{1}}{\partial t_{1}} \ldots \frac{\partial \varphi_{1}}{\partial t_{n}} \\
\cdots \cdots \\
\frac{\partial \varphi_{n-1}}{\partial t_{1}} \ldots \frac{\partial \dot{\varphi}_{n-1}}{\partial t_{n}}
\end{array}\right| \equiv 0 \bmod \partial .
$$

\section{$\S 3$ GENERATORS OF THE MULTIPLICATIVE GROUP $F^{*}$}

We say that a unit $\varepsilon$ of the field $F$ is principal if its image in the residue field $k_{0}$ is 1 . The set of all the principal units forms a group.

If we fix a prime element $\pi$ and local parameters $t_{1}, \ldots, t_{n-1}$ of the field $F$, then any $a \in F^{*}$ can be represented as a formal power series on $t_{1}, \ldots, t_{n-1}, \pi$ with coefficients from the multiplicative element system $\Re$ of the field $k_{0}$. In such a representation there always exists a term $\theta t_{1}^{i_{1}} \ldots t_{n-1}^{i_{n-1}} \pi^{i_{n}}, \theta \in \mathfrak{R}$, with the minimal (in the lexicographical order) set of exponents $\left(i_{1}, \ldots, i_{n}\right)$. This will be denoted by a congruence:

$$
a \equiv \theta t_{1}^{i_{1}} \cdot \ldots \cdot t_{n-1}^{i_{n-1}} \pi^{i_{n}} \quad \bmod \operatorname{deg}\left(i_{1}, \ldots, i_{n}\right) .
$$

In particular:

$$
2 \equiv \theta_{2} t_{1}^{e_{1}} \cdot \ldots \cdot t_{n-1}^{e_{n-1}} \pi^{e_{n}} \bmod \operatorname{deg}\left(e_{1}, \ldots, e_{n}\right)
$$

where $e_{1}, \ldots, e_{n}$ are the ramification indices in the extensions $k_{1} / k_{0}, \ldots k_{n} / k_{n-1}$ respectivly.

Any element $\alpha$ of $F^{*}$ has the following representation:

$$
\alpha=t_{1}^{a_{1}} \cdot \ldots \cdot t_{n-1}^{a_{n-1}} \pi^{a_{n}} \theta \varepsilon, a_{i} \in \mathbb{Z}, \theta \in \mathfrak{R},
$$

where $\varepsilon$ is a principal unit. 
Any principal unit of $F^{*}$ may be written in the form:

$$
\varepsilon=1+a t_{1}^{i_{1}} \cdot \ldots \cdot t_{n-1}^{i_{n-1}} \pi^{i_{n}}, a \in A, i_{n} \geq 0, i_{1}, \ldots, i_{n-1} \in \mathbb{Z},
$$

and if $i_{n}=0$, then the last non-zero $i_{r}$ is to be positive.

Suppose $\varepsilon \equiv 1-\theta t_{1}^{i_{1}} \ldots \ldots t_{n-1}^{i_{n-1}} \pi^{i_{n}} \bmod \operatorname{deg}\left(i_{1}, \ldots, i_{n}\right), \theta \in \mathfrak{R}$.

Consider the following cases:

a) $\left(i_{1}, \ldots, i_{n}\right)<\left(e_{1}, \ldots, e_{n}\right)$ in the lexicographical order, then

$$
\varepsilon^{2} \equiv 1-\theta^{2} t_{1}^{2 i_{3}} \cdot \ldots \cdot t_{n-1}^{2 i_{n-1}} \pi^{2 i_{n}} \quad \bmod \operatorname{deg}\left(2 i_{1}, \ldots, 2 i_{n}\right)
$$

b) $\left(i_{1}, \ldots, i_{n}\right)>\left(e_{1}, \ldots, e_{n}\right)$, then

$$
\varepsilon^{2} \equiv 1-\theta_{2} t_{1}^{i_{1}+e_{1}} \cdot \ldots \cdot t_{n-1}^{i_{n-1}+e_{n-1}} \pi^{i_{n}+e_{n}} \bmod \operatorname{deg}\left(i_{1}+e_{1}, \ldots, i_{n}+e_{n}\right) ;
$$

c) $\left(i_{1}, \ldots, i_{n}\right)=\left(e_{1}, \ldots, e_{n}\right)$, then

$$
\varepsilon^{2} \equiv 1-\left(\theta_{2} \theta+\theta^{2}\right) t_{1}^{2 e_{1}} \cdot \ldots \cdot t_{n-1}^{2 e_{n-1}} \pi^{2 e_{n}} \bmod \operatorname{deg}\left(2 e_{1}, \ldots, 2 e_{n}\right) .
$$

From these congruences, using the standard method of [4], we obtain the following set of generators in the group of the principal units:

$$
\varepsilon_{c, i}=1-c \pi^{i}, \quad 0 \leq i \leq 2 e_{n} .
$$

Here we denote by $c$ the product $\theta t_{1}^{i_{1}} \cdot \ldots \cdot t_{n-1}^{i_{n-1}}$, where $\theta \in \mathfrak{A}$ and $i_{1}, \ldots, i_{n-1}, i$ satisfy:

a) $i_{1}, \ldots, i_{n-1, i} \in \mathbb{Z}$

b) among $i_{1}, \ldots, i_{n-1}, i$ there exists an odd number;

c) the last non-zero $i_{r}$ before $i$ is positive if $i=0$ and less then $2 e_{r}$ if $i=2 e_{n}$.

We must also add a $q$-prime element

$$
\omega_{*}=\left.E(\xi s(X))\right|_{X=\pi}, \operatorname{tr} \xi \equiv 1 \bmod q .
$$

Thus we get the following set of generators:

$$
\left\{\varepsilon_{c, i}, \omega_{*}\right\}, \quad 0 \leq i \leq 2 e_{n} .
$$

By the definition of the function $E$ for the elements $\rho_{c, i}=E\left(c \pi^{i}\right)$ we obtain the following congruence

$$
\rho_{c, i} \equiv 1-c \pi^{i} \bmod \left(c \pi^{i}\right)^{2} .
$$

So we may take for the set of generators the set

$$
\left\{\rho_{c, i}, \omega_{*}\right\}, \quad 0 \leq i \leq 2 e_{n},
$$

with the same conditions on the indices. 


\section{$\S 4$ THE PROPERTIES OF THE MAP $\Gamma$.}

In this part we deal with the proof of the multiplicativity, the anticommutativity, the proportionality and the symbol property of the map $\Gamma$, stated in the introduction (Theorem 1).

Multiplicativity is clear because of the obvious $(\forall 1 \leq i \leq n)$ relatoins:

$$
\begin{aligned}
& \delta_{i}(\alpha \beta)=\delta_{i}(\alpha)+\delta_{i}(\beta), \\
& \eta_{i}(\alpha \beta)=\eta_{i}(\alpha)+\eta_{i}(\beta), \\
& \sigma_{i}(\alpha \beta)=\sigma_{i}(\alpha)+\sigma_{i}(\beta) .
\end{aligned}
$$

To prove the anticommutativity, by the Lemma 2, it is enough to verify the congruence:

$$
\Phi\left(\ldots, \alpha_{i}, \alpha_{i+1} \ldots\right)+\Phi\left(\ldots, \alpha_{n+1}, \alpha_{i}, \ldots\right) \equiv 0 \quad \bmod \partial .
$$

For sinplicity the proof will be given for the first pair of elements, i.e. we will prove the songruence:

$$
\Phi\left(\alpha_{1}, \alpha_{2}, \ldots, \alpha_{n+1}\right)+\Phi\left(\alpha_{2}, \alpha_{1}, \ldots, \alpha_{n+1}\right) \equiv 0 \quad \bmod \partial
$$

Note that after expansion of all the determinants in the definition of the series $\Phi^{(1)}$, except $D_{i+1}$, with respect to the last row we obtain an equality (see [1]):

$\left.{ }^{*}\right) \quad \Phi^{(1)}\left(\alpha_{1}, \ldots, \alpha_{n+1}\right)=l\left(\alpha_{n+1}\right) D_{n+1}+\sum_{i=1}^{n}(-1)^{n+i+1} \eta_{i}\left(\alpha_{n+1}\right) \Phi_{i}\left(\alpha_{1}, \ldots, \alpha_{n}\right)$

where $\Phi_{i}$ are the power series of $n-1$ variables $t_{1}, \ldots, t_{i-1}, t_{i+1}, \ldots, t_{n}$ with the coefficients in $o\left\{\left\{t_{i}\right\}\right\}$, given by the very same formula as $\Phi^{(1)}$.

The proof is by induction.

For $n=1$ we have:

$$
\Phi^{(1)}\left(\alpha_{1}, \alpha_{2}\right)+\Phi^{(1)}\left(\alpha_{2}, \alpha_{1}\right)=\frac{\partial}{\partial t} l\left(\alpha_{1}\right) l\left(\alpha_{2}\right) \equiv 0 \bmod \partial
$$

Assume that our congruence holds for $n-1$.

Representing $\Phi^{(1)}\left(\alpha_{1}, \alpha_{2}, \ldots, \alpha_{n+1}\right)$ and $\Phi^{(1)}\left(\alpha_{2}, \alpha_{1}, \ldots, \alpha_{n+1}\right)$ as in $\left(^{*}\right)$ and taking into consideration the induction hypothesis for the series $\Phi_{i}$ we obtain:

$$
\begin{gathered}
\Phi^{(1)}\left(\alpha_{1}, \alpha_{2}, \ldots, \alpha_{n+1}\right)+\Phi^{(1)}\left(\alpha_{2}, \alpha_{1}, \ldots, \alpha_{n+1}\right) \equiv l\left(\alpha_{n+1}\right)\left|\begin{array}{c}
\delta_{1}\left(\alpha_{1}\right) \ldots \delta_{n}\left(\alpha_{1}\right) \\
\delta_{1}\left(\alpha_{2}\right) \ldots \delta_{n}\left(\alpha_{2}\right) \\
\ldots \ldots \ldots \\
\delta_{1}\left(\alpha_{n}\right) \ldots \delta_{n}\left(\alpha_{n}\right)
\end{array}\right| \\
+l\left(\alpha_{n+1}\right)\left|\begin{array}{c}
\delta_{1}\left(\alpha_{2}\right) \ldots \delta_{n}\left(\alpha_{2}\right) \\
\delta_{1}\left(\alpha_{1}\right) \ldots \delta_{n}\left(\alpha_{1}\right) \\
\ldots \ldots \ldots \\
\delta_{1}\left(\alpha_{n}\right) \ldots \delta_{n}\left(\alpha_{n}\right)
\end{array}\right|=0 \bmod \partial
\end{gathered}
$$


Consider now $\Phi^{(2)}\left(\alpha_{1}, \alpha_{2}, \ldots, \alpha_{n+1}\right)+\Phi^{(2)}\left(\alpha_{2}, \alpha_{1}, \ldots, \alpha_{n+1}\right)$.

It is easy to see that for $i, j \notin\{1 ; 2\}$ the interchange of $\alpha_{1}$ and $\alpha_{2}$ means only an interchange of two adjacent lines in $P_{i, j}$, and $P_{i, 1}$ becomes $P_{i, 2}$ and vice versa. So we obtain

$$
\begin{aligned}
& \Phi^{(2)}\left(\alpha_{1}, \alpha_{2}, \ldots, \alpha_{n+1}\right)+\Phi^{(2)}\left(\alpha_{2}, \alpha_{1}, \ldots, \alpha_{n+1}\right)= \\
& =2 P_{2,1}+2 \sum_{i=3}^{n+1}\left(P_{i, 1}+P_{i, 2)} \equiv 0 \bmod \partial,\right.
\end{aligned}
$$

since $2 \nu_{l}\left(\alpha_{1}, \alpha_{2}\right) \equiv 0 \bmod \partial, \quad \forall 1 \leq l \leq n$.

Thus $\Phi\left(\alpha_{1}, \alpha_{2}, \ldots, \alpha_{n+1}\right)+\Phi\left(\alpha_{2}, \alpha_{1}, \ldots, \alpha_{n+1}\right) \equiv 0 \bmod \partial$, q.e.d.

The symbol property means an equality:

$$
\Gamma(\ldots, \alpha, 1-\alpha, \ldots)=1 .
$$

Because of the multiplicativity, anticommutativity and Lemma 1 it is enough to prove the congruence:

$$
\Phi\left(t_{1}, \ldots, t_{n-1}, \alpha, 1-\alpha\right) \equiv 0 \quad \bmod \partial
$$

Taking into consideration that $l\left(t_{i}\right)=0$ we obtain:

$$
\begin{aligned}
& \Phi\left(t_{1}, \ldots, t_{n-1}, \alpha, 1-\alpha\right)=l(1-\alpha)\left|\begin{array}{c}
\delta_{1}\left(t_{1}\right) \ldots \delta_{n}\left(t_{1}\right) \\
\ldots \ldots \ldots \\
\delta_{1}\left(t_{n-1}\right) \ldots \delta_{n}\left(t_{n-1}\right) \\
\delta_{1}(\alpha) \ldots \delta_{n}(\alpha)
\end{array}\right|- \\
& -l(\alpha)\left|\begin{array}{c}
\delta_{1}\left(t_{1}\right) \ldots \delta_{n}\left(t_{1}\right) \\
\ldots \ldots \ldots \\
\delta_{1}\left(t_{1}\right) \ldots \delta_{n}\left(t_{1}\right) \\
\ldots \ldots \ldots \\
\delta_{1}(1-\alpha) \ldots \delta_{n}\left(t_{n-1}\right) \\
\delta_{1}\left(t_{n-1}\right) \ldots \delta_{n}\left(t_{n-1}\right) \\
\nu_{1}(\alpha, 1-\alpha) \ldots \nu_{n}(\alpha, 1-\alpha)
\end{array}\right|= \\
& =t_{1}^{-1} \ldots t_{n-1}^{-1} \sum_{i=1}^{n}\left(l(1-\alpha) \delta_{i}(\alpha)-l(\alpha) \eta_{i}(1-\alpha)+\frac{\partial}{\partial t_{i}} \frac{\Delta}{2} \sigma(\alpha) \sigma(1-\alpha)\right)
\end{aligned}
$$

i.e. we come to the 1-dimensional case proved in [3].

Proportionality means the equality

$$
\Gamma(\ldots, \alpha,-\alpha, \ldots)=1
$$

and it follows from the other three properties:

$$
\begin{aligned}
1=\Gamma(\ldots, & \left.\frac{1}{\alpha}, 1-\frac{1}{\alpha}, \ldots\right)=\Gamma\left(\ldots, \alpha, 1-\frac{1}{\alpha}, \ldots\right)^{-1}= \\
& =\Gamma(\ldots, \alpha,-\alpha, \ldots)^{-1} \Gamma(\ldots, \alpha, 1-\alpha, \ldots)^{-1}=\Gamma(\ldots, \alpha,-\alpha, \ldots)^{-1} .
\end{aligned}
$$




\section{$\S 5$ INDEPENDENCE AND INVARIANCE OF THE MAP $\Gamma$.}

This part contains the proof of Theorem 2. We shall reduce the independence and invariance to the 1-dimensional case.

Independence of the map $\Gamma$ is equivalent to following: if an element $\alpha_{i}$ of $F^{*}$ is decomposed in the series on $t_{1}, \ldots, t_{n-1}$ and the prime $\pi$ in two different ways and the series $\varepsilon\left(t_{n}\right)$ is obtained as a quotient of these two series (after replacement of $\pi$ by $t_{n}$ ), then the congruence

$$
\operatorname{tr} \operatorname{res} \Phi\left(\alpha_{1}, \ldots, \varepsilon, \ldots, \alpha_{n+1}\right) \cdot W \equiv 0 \quad \bmod q
$$

holds at any $\alpha_{1}, \ldots, \alpha_{i-1}, \alpha_{i+1}, \ldots, \alpha_{n+1} \in A\left\{\left\{t_{n}\right\}\right\}$.

It suffices to show that

$$
\operatorname{tr} \text { res } \Phi\left(t_{1}, \ldots, \ldots, t_{n-1}, \pi, \varepsilon,\right) \cdot W \equiv 0 \quad \bmod q .
$$

Take into into account that $l\left(t_{i}\right)=l(\pi)=0$ and $\delta\left(t_{i}\right)=\delta(\pi)=0$. Replacing $\pi$ by $t_{n}$ we obtain:

$$
\Phi\left(t_{1}, \ldots, \ldots, t_{n}, \varepsilon,\right)=t_{1}^{-1} \cdot \ldots \cdot t_{n-1}^{-1} t_{n}^{-1} l(\varepsilon) .
$$

Thus we have to prove the congruence:

$$
\text { tr res } t_{1}^{-1} \cdot \ldots \cdot t_{n-1}^{-1}\left(t_{n}^{-1} l(\varepsilon) \cdot W\right) \equiv 0 \quad \bmod q,
$$

and this is a 1-dimensional case.

Let now $x_{1}, \ldots, x_{n}$ and $t_{1}, \ldots, t_{n}$ be two different sets of uniformizing elements. Then the invariance of the map $\Gamma$ means, that if there is a change of variables:

$$
\begin{gathered}
x_{1} \mapsto t_{1}, \\
\ldots \\
x_{n} \mapsto t_{n},
\end{gathered}
$$

then $\Gamma_{x}\left(\alpha_{1}, \ldots, \alpha_{n+1}\right)=\Gamma_{t}\left(\alpha_{1}, \ldots, \alpha_{n+1}\right)$

Because of the independence it is enough to verify the invariance for changes of the following type:

$$
\begin{gathered}
x_{1}=t_{1}, \\
\ldots \\
x_{i-1}=t_{i-1}, \\
x_{i}=g\left(t_{1}, \ldots, t_{n}\right), \\
x_{i+1}=t_{i+1}, \\
\ldots \\
x_{n}=t_{n}
\end{gathered}
$$


Because of the multiplicativity and anticommutativity of $\Gamma$ it suffices to show that

$$
\Gamma_{x}\left(x_{1}, \ldots, x_{n}, \mathcal{E}\left(x_{1}^{i_{1}} \cdot \ldots \cdot x_{n-1}^{i_{n-1}} x_{n}^{k}\right)=\Gamma_{t}\left(t_{1}, \ldots, t_{n-1}, g, \mathcal{E}\left(t_{1}^{i_{1}} \cdot \ldots \cdot t_{n-1}^{i_{n-1}} g^{k}\right)\right)\right.
$$

To prove this equality it is sufficient to verify the congruence:

$\Phi_{x}\left(x_{1}, \ldots, x_{n}, \mathcal{E}\left(x_{1}^{i_{1}} \ldots x_{n-1}^{i_{n-1}} x_{n}^{k}\right)\right) \equiv \Phi_{t}\left(t_{1}, \ldots, t_{n-1}, g, \mathcal{E}\left(t_{1}^{i_{1}} \ldots . t_{n-1}^{i_{n-1}} g^{k}\right)\right) \quad \bmod \partial$

Denoting $x_{1}^{i_{1}} \cdot \ldots \cdot x_{n-1}^{i_{n-1}}=t_{1}^{i_{1}} \cdot \ldots \cdot t_{n-1}^{i_{n-1}}$ by $c$ and taking into consideration that $l(x)=0$ for any uniformizing $x$, we obtain:

$$
\begin{gathered}
\Phi_{x}\left(x_{1}, \ldots, x_{n}, \mathcal{E}\left(c x_{n}^{k}\right)\right)=l\left(\mathcal{E}\left(c x_{n}^{k}\right)\right)\left|\begin{array}{c}
\delta_{1}\left(x_{1}\right) \ldots \delta_{n}\left(x_{1}\right) \\
\ldots \ldots \ldots \\
\delta_{1}\left(x_{n}\right) \ldots \delta_{n}\left(x_{n}\right)
\end{array}\right|=c x_{1}^{-1} \ldots x_{n-1}^{-1} x_{n}^{k-1} \\
\Phi_{t}\left(t_{1}, \ldots, t_{n-1}, g, \mathcal{E}\left(t_{1}^{i_{1}} \ldots t_{n-1}^{i_{n-1}} g^{k}\right)\right)=l\left(\mathcal{E}\left(c g^{k}\right)\right)\left|\begin{array}{c}
\delta_{1}\left(t_{1}\right) \ldots \delta_{n}\left(t_{1}\right) \\
\ldots \ldots \ldots \\
\delta_{1}(g) \ldots \delta_{n}(g)
\end{array}\right|- \\
-l(g)\left|\begin{array}{c}
\delta_{1}\left(t_{1}\right) \ldots \delta_{n}\left(t_{1}\right) \\
\delta_{1}\left(t_{1}\right) \ldots \delta_{n}\left(t_{1}\right) \\
\eta_{1}\left(\mathcal{E}\left(c g^{k}\right)\right) \ldots \eta_{n}\left(\mathcal{E}\left(c g^{k}\right)\right)
\end{array}\right|+\left|\begin{array}{c}
\ldots \\
\nu_{1}\left(g, \mathcal{E}\left(c g^{k}\right)\right) \ldots \nu_{n}\left(g, \mathcal{E}\left(c g^{k}\right)\right)
\end{array}\right|= \\
=\sum_{i=1}^{n} t_{1}^{-1} \ldots \ldots t_{n-1}^{-1}\left(l\left(\mathcal{E}\left(c g^{k}\right)\right) \delta_{i}(g)-l(g) \eta_{i}\left(\mathcal{E}\left(c g^{k}\right)\right)+\nu_{i}\left(g, \mathcal{E}\left(c g^{k}\right)\right)\right) .
\end{gathered}
$$

So, it is enough to prove that

$$
c x_{n}^{k-1} \equiv l\left(\mathcal{E}\left(c g^{k}\right)\right) \delta_{i}(g)-l(g) \eta_{i}\left(\mathcal{E}\left(c g^{k}\right)\right)+\nu_{i}\left(g, \mathcal{E}\left(c g^{k}\right)\right) \quad \bmod \partial
$$

and once again we come to the 1-dimensional case (see [2.2])

$\S 6$ CoIncidence of the PaIRING $<,>_{\Gamma}$ With the Hilbert symbol.

To prove the coincidence of the pairing $<,>_{\Gamma}$ with the Hilbert symbol $(,)_{q}$ it is enough to verify the coincidence of their values on the pairs $\left(x_{\pi}, \varepsilon\right)$, where $x_{\pi}=\left\{t_{1}, \ldots, t_{n-1}, \pi\right\}$, and $\varepsilon$ is a principal unit of the field $F$ (see [1] for more details). It is clear that it suffices to take for $\varepsilon$ only the generators of the group of principal units (see $\S 3$ ), i.e. it is enough to consider the two following cases:

a) $\varepsilon=\omega_{*}$,

b) $\varepsilon=\varepsilon_{c, i}$

By the definition of the Hilbert symbol we obtain an equality:

$$
\left(x_{\pi}, \omega_{*}\right)_{q}=\zeta
$$


As defined above $\varepsilon_{c, i}=1-c \pi^{i}=1-t_{1}^{i_{1}} \ldots \cdot t_{n-1}^{i_{n-1}} \pi^{i}$ where $\theta \in \Re$ and at least one of $i_{1}, \ldots, i_{n-1}, i$ is an odd number. Assume that $i$ is odd. Then we have:

$$
\left(x_{\pi}, \varepsilon_{c, i}\right)_{q}^{i}=\left(\left\{t_{1}, \ldots, t_{n-1}, c \pi^{i}\right\}, \varepsilon_{c, i}\right)_{q}\left(\left\{t_{1}, \ldots, t_{n-1}, c\right\}, \varepsilon_{c, i}\right)_{q}^{-1} .
$$

The first of these factors is trivial by the symbol property, and the second one is trivial by the multiplicativity and proportionality. Thus $\left(x_{\pi}, \varepsilon_{c, i}\right)_{q}=1$.

The case when the odd number is one of $i_{1}, \ldots, i_{n-1}$ is similar.

By the definition of the pairing \langle\rangle$_{\Gamma}$ and $\omega_{*}$ we obtain:

$$
<x_{\pi}, \omega_{*}>_{\Gamma}=\zeta
$$

The pairing \langle\rangle$_{\Gamma}$ has the very same properties as the Hilbert symbol, so we can prove, as shown above for the Hilbert symbol, the following equality:

$$
<x_{\pi}, \varepsilon_{c, i}>_{\Gamma}=1 \text {. }
$$

Thus the pairing $\left\langle>>_{\Gamma}\right.$ and the Hilbert symbol coincide at the generators, and, consequently, everywhere.

\section{BIBLIOGRAPHY}

1. Vostokov,S.V., Explicit construction of class field theory for a multidimensional local field, Math USSR, Izv. 26 (1986), 263-287.

2.1. Vostokov,S.V., The Hilbert symbol for Lubin-Tate formal groups. I, J. Sov. Math. 27 (1984), 2885-2901.

2.2. Vostokov,S.V.; Fesenko,I.B., The Hilbert symbal for Lubin-Tate formal groups. II, J. Sov. Math. 30 (1985), 1854-1862.

3. Henniart,G., Sur les lois de réciprocité explicites. I, J. Reine Angew. Math. 329 (1981), 177-203.

4. Shafarevich, I.R., A general reciprocity law, Amer. Math. Soc. Transl. (2) 4 (1956), 73-106. 
$\$ 1$.

The correct formulas for $D_{i}$ and $P_{i, j}$ are:

$$
D_{i}=\left|\begin{array}{ccc}
\delta_{1}\left(\alpha_{1}\right) & \ldots & \delta_{n}\left(\alpha_{1}\right) \\
\ldots & \ldots & \ldots \\
\delta_{1}\left(\alpha_{i-1}\right) & \ldots & \delta_{n}\left(\alpha_{i-1}\right) \\
\eta_{1}\left(\alpha_{i+1}\right) & \ldots & \eta_{n}\left(\alpha_{i+1}\right) \\
\ldots & \ldots & \ldots \\
\eta_{1}\left(\alpha_{n+1}\right) & \ldots & \eta_{n}\left(\alpha_{n+1}\right)
\end{array}\right|, P_{i, j}=\left|\begin{array}{ccc}
\delta_{1}\left(\alpha_{1}\right) & \ldots & \delta_{n}\left(\alpha_{1}\right) \\
\ldots & \ldots & \ldots \\
\delta_{1}\left(\alpha_{j-1}\right) & \ldots & \delta_{n}\left(\alpha_{j-1}\right) \\
\nu_{1}\left(\alpha_{j}, \alpha_{i}\right) & \ldots & \nu_{n}\left(\alpha_{j}, \alpha_{i}\right) \\
\delta_{1}\left(\alpha_{j+1}\right) & \ldots & \delta_{n}\left(\alpha_{j+1}\right) \\
\ldots & \ldots & \ldots \\
\delta_{1}\left(\alpha_{i-1}\right) & \ldots & \delta_{n}\left(\alpha_{i-1}\right) \\
\eta_{1}\left(\alpha_{i+1}\right) & \ldots & \eta_{n}\left(\alpha_{i+1}\right) \\
\ldots & \ldots & \ldots \\
\eta_{1}\left(\alpha_{n+1}\right) & \ldots & \eta_{n}\left(\alpha_{n+1}\right)
\end{array}\right|
$$

$\$ 4$.

In the poof of the symbol property the words "Because of the multiplicativity, anticommutativity and Lemma 1 it is enough to prove the congruence:" should be replaced by "We shall prove this for the case, when all the elements except $\alpha$ and $1-\alpha$ are uniformizing elements. So it is enough to verify the congruence:"

Because of this remark the proof in this article is complete only for the case, when our map is defined on the elements $\left\{t-1, \ldots, t_{n}, \alpha, \varepsilon\right\}$, where $\alpha \in A\left\{\left\{t_{n}\right\}\right\}$ and $\varepsilon$ is a unit. Knowing the values of the Hilbert symbol on these elements, one can calculate its value on any other element. The more complete proof will be given in the next article.

We are deeply grateful to Professor I.B.Fesenko for his useful remarks and attention to this work. 